PROCEEDINGS OF THE

AMERICAN MATHEMATICAL SOCIETY

Volume 139, Number 1, January 2011, Pages 375-382

S 0002-9939(2010)10555-5

Article electronically published on August 5, 2010

\title{
TWO QUESTIONS ON MAPPING CLASS GROUPS
}

\author{
LOUIS FUNAR
}

(Communicated by Daniel Ruberman)

\begin{abstract}
We show that central extensions of the mapping class group $M_{g}$ of the closed orientable surface of genus $g$ by $\mathbb{Z}$ are residually finite. Further we give rough estimates of the largest $N=N_{g}$ such that homomorphisms from $M_{g}$ to $S U(N)$ have finite image. In particular, homomorphisms of $M_{g}$ into $S L([\sqrt{g+1}], \mathbb{C})$ have finite image. Both results come from properties of quantum representations of mapping class groups.
\end{abstract}

\section{INTRODUCTION AND STATEMENTS}

Set $\Sigma_{g}^{r}$ for the orientable surface of genus $g$ with $r$ punctures. We denote by $M_{g}^{r}$ the mapping class group of $\Sigma_{g}^{r}$, namely the group of isotopy classes of homeomorphisms that fix the punctures.

The following answers Question 6.4 of Farb (see Chapter 2 of [7]).

Proposition 1.1. The central extensions of the mapping class group $M_{g}$ (or the punctured mapping class group $M_{g}^{1}$, for $g \geq 4$ ) by $\mathbb{Z}$ are residually finite.

Remark 1.1. The universal central extension $\widetilde{M}_{g}(1)$ surjects onto the universal central extension $S \widetilde{p(2 g, \mathbb{Z})}$ of the (integral) symplectic group, whose class is the Maslov class (generating $H^{2}(S p(2 g, \mathbb{Z}))$. It is known that $\widehat{S p(2 g, \mathbb{Z})}$ is the pull-back of $S p(2 g, \mathbb{Z})$ into the universal covering $S \widetilde{S p(2 g, \mathbb{R})}$ of the real symplectic group.

By a result of Deligne (see [6]) the extension $S p(2 g, \mathbb{Z})$, for $g \geq 2$, is not residually finite since any finite index subgroup of it contains $2 \mathbb{Z}$, where $\mathbb{Z}$ is the central kernel $\widehat{S p(2 g, \mathbb{Z})} \rightarrow S p(2 g, \mathbb{Z})$. The same holds, more generally, for some other arithmetic groups having the congruence subgroup property.

The method of proof uses quantum representations of mapping class groups.

Definition 1.1. The group $\Gamma$ has property $F_{n}$ if all homomorphisms $\Gamma \rightarrow P U(n)$ have finite image. Moreover, the group $\Gamma$ has property $F$ if it has property $F$ for every $n$.

Observe that the property $F$ is inherited by finite index subgroups.

Remark 1.2. Let $G$ be a connected, semi-simple, almost $\mathbb{Q}$-simple algebraic $\mathbb{Q}$-group and $\Gamma$ an arithmetic lattice in $G$. If $G_{\mathbb{R}}$ has real rank at least 2 and $G^{a d}(\mathbb{R})$ has

Received by the editors October 12, 2009 and, in revised form, April 2, 2010.

2010 Mathematics Subject Classification. Primary 57M07, 20F36, 20F38, 57N05.

Key words and phrases. Mapping class group, central extension, quantum representation.

(C)2010 American Mathematical Society 
no compact factor, then $\Gamma$ has property $F$. This follows from (17], Chap. VIII, Thm. B) for $K=\mathbb{Q}, l=\mathbb{R}, S$ containing only the Archimedean place of $\mathbb{Q}$ and $\mathbf{H}=P O(n)$. In particular, any discrete group $\Gamma$ commensurable with $S p(2 g, \mathbb{Z})$ for $g \geq 2$ or to $S L(2, \mathcal{O})$, where $\mathcal{O}$ is the ring of integers in a totally real number field of degree at least 2 , has property $F$.

Mapping class groups do not have property $F$. It is therefore interesting to understand whether they have property $F_{n}$ for some $n$. This is related to a question of Farb in [7 concerning linear representations in small degree. The previous remark shows that we cannot use unitary representations of $M_{g}$ that factor through $S p(2 g, \mathbb{Z})$, as the latter group has no finite-dimensional unitary representations with infinite image. Our second result is stated as follows:

Proposition 1.2. The maximal number $N_{g}$ for which $M_{g}$ has property $F_{N_{g}}$ satisfies

$$
\sqrt{g+1} \leq N_{g}<\left\{\begin{array}{l}
5^{g / 2} F_{g-1}, \text { if } g \text { is even, } \\
5^{(g-1) / 2}\left(F_{g}+F_{g-2}\right), \text { if } g \text { is odd, }
\end{array}\right.
$$

where $F_{j}$ is the Fibonacci sequence, defined by $F_{0}=0, F_{1}=1$ and the recurrence $F_{n+1}=F_{n}+F_{n-1}$, for $n \geq 1$. Moreover, the upper bounds are valid for any finite index subgroup of $M_{g}$.

Corollary 1.1. Every homomorphism $M_{g} \rightarrow S L([\sqrt{g+1}], \mathbb{C})$ has finite image if $g \geq 1$.

It is likely that $N_{g}$ behaves like an exponential for large $g$. This seems difficult to check because very few unitary representations of $M_{g}$ are known. On the other hand, one might expect that the maximal $n$ with the property that every homomorphism $M_{g} \rightarrow S L(n, \mathbb{C})$ has finite image is a linear function on $g$.

Notice that groups having homomorphisms with infinite image into $S L(2, \mathbb{C})$ do not have the property $T$ of Kazhdan. However, $M_{g}$ has no such representations if $g \geq 3$, by the corollary above.

Results of a similar flavor were proved in 10, where it is shown that representations $M_{g} \rightarrow G L(2 \sqrt{g-1}, \mathbb{C})$ cannot be faithful and in [3, where it is shown that the image of an element of $M_{g}$ under a representation into $G L(g, \mathbb{C})$ should have algebraic eigenvalues.

One inequality above is an immediate consequence of a theorem of Bridson ([3]) concerning the property $F A_{n}$, which was introduced by Farb in 9 . The second inequality comes from the existence of quantum representations of $M_{g}$ with infinite image $([13])$.

\section{Proof of Proposition 1.1}

We prove the claim for the universal central extension first. This is known when $g=1$ since the universal central extension is isomorphic to the braid group $B_{3}$.

An important result due independently to Andersen ([1]) and to Freedman, Walker and Wang ([1]) states that the $S U(2)$ TQFT representation of the mapping class group is asymptotically faithful. Specifically, there is a sequence of representations $\rho_{k}$ (indexed by an integer $k$, called the level) $\rho_{k}: M_{g} \rightarrow P U(N(k, g))$ into the projective unitary group of dimension $N(k, g)$ (for some $N(k, g)$ depending exponentially on $k$ ) such that the intersection of the kernels $\bigcap_{k>2} \operatorname{ker} \rho_{k}$ is trivial for $g \geq 3$, and respectively the center of the mapping class group $\bar{M}_{2}$ (which is a group of order two generated by hyperelliptic involution) when $g=2$. Moreover, for $g=2$ 
we can use the $S U(n)$ TQFT representation, with $n \geq 3$, for which the intersection of the kernels above is trivial (see [1]). When using this result we will say that we make use of the asymptotic faithfulness (of the quantum representations).

Each quantum representation is a projective representation which lifts to a linear representation $\widetilde{\rho}_{k}: \widetilde{M}_{g}(12) \rightarrow U(N(k, g))$ of the central extension $\widetilde{M}_{g}(12)$ of the mapping class group $M_{g}$ by $\mathbb{Z}$. The latter representation corresponds to invariants of 3-manifolds with a $p_{1}$-structure. Masbaum and Roberts ([18]) and Gervais ([15]) gave a precise description of this extension. Namely, the cohomology class $c_{\widetilde{M}_{g}(12)} \in$ $H^{2}\left(M_{g}, \mathbb{Z}\right)$ associated to this extension equals 12 times the signature class $\chi$. It is known (see [16]) that the group $H^{2}\left(M_{g}\right)$ is generated by $\chi$ when $g \geq 2$. Recall that $\chi$ is the class of one fourth the Meyer signature cocycle.

Observe that the $\rho_{k}$ action of the center of $\widetilde{M}_{g}(12)$ is by roots of unity of order $2 k$ (see [18] for the explicit formula). In fact, this action corresponds to the change of the $p_{1}$-structure of a 3 -manifold, and it is well-known that the quantum invariant changes by a root of unity of order $2 k$. Thus every element of the center acts nontrivially via $\widetilde{\rho}_{k}$, for large enough $k$, so that the representations of $\widetilde{M}_{g}(12)$ are also asymptotically faithful. This implies that $\widetilde{M}_{g}(12)$ is residually finite. In fact, let $a \in \widetilde{M}_{g}(12)$ be any element $a \neq 1$. By the asymptotic faithfulness there exists some level $k$ so that $\widetilde{\rho}_{k}(a) \in U(N(k, g))$ is non-trivial. The subgroup $\widetilde{\rho}_{k}\left(\widetilde{M}_{g}(12)\right) \subset$ $U(N(k, g))$ is a discrete linear group and thus, by a classical theorem of Malcev, it is residually finite. In particular, there exists a homomorphism of $\widetilde{\rho}_{k}\left(\widetilde{M}_{g}(12)\right)$ onto some finite group sending $\widetilde{\rho}_{k}(a)$ into a non-trivial element. This shows that every non-trivial element of $\widetilde{M}_{g}(12)$ is detected by some homomorphism into some finite group.

The universal central extension is $\widetilde{M}_{g}(1)$, where $\widetilde{M}_{g}(n)$ denotes the central extension by $\mathbb{Z}$ whose class is $c_{\widetilde{M}_{g}(n)}=n \chi$. It is immediate from their explicit presentations (see [15]) that $\widetilde{M}_{g}(d)$ embeds into $\widetilde{M}_{g}(n)$ if $d$ divides $n \neq 0$. Such an embedding sends the generator $z$ of the center into $z^{n / d}$. In particular, $\widetilde{M}_{g}(1)$ embeds in $\widetilde{M}_{g}(12)$, and thus the universal central extension is residually finite.

Now, an arbitrary central extension of $M_{g}$ by $\mathbb{Z}$ is either trivial and hence residually finite or else isomorphic to $\widetilde{M}_{g}(n)$, for some $n \in \mathbb{Z} \backslash\{0\}$. We observed above that there is an injective homomorphism $\widetilde{M}_{g}(1) \rightarrow \widetilde{M}_{g}(n)$, which sends the central element $z$ into $z^{n}$. Moreover, the image is a normal subgroup of $\widetilde{M}_{g}(n)$. In particular, we have $\widetilde{M}_{g}(n) / \widetilde{M}_{g}(1)=\mathbb{Z} / n \mathbb{Z}$. This implies that $\widetilde{M}_{g}(n)$ is residually finite. In fact, any element of $\widetilde{M}_{g}(n)$ which is not detected by the homomorphism onto $\mathbb{Z} / n \mathbb{Z}$ belongs to $\widetilde{M}_{g}(1)$. Inducting finite group representations from $\widetilde{M}_{g}(1)$ to $\widetilde{M}_{g}(n)$, we obtain finite group representations of the latter detecting every non-trivial element of $\widetilde{M}_{g}(1)$. This proves the claim.

Remark 2.1. Freedman, Walker and Wang observed in [11] that a simple consequence of the asymptotic faithfulness is that $M_{g}$ is residually finite.

Remark 2.2. This proof works more generally for the punctured mapping class group $M_{g}^{1}$ and for those extensions whose cohomology classes are of the form $n \chi+e$, for some $n \in \mathbb{Z}$. Recall that $H^{2}\left(M_{g}^{1}\right)=\mathbb{Z} \chi \oplus \mathbb{Z} e$, where $\chi$ is the signature class and $e$ is the class associated to the puncture, for $g \geq 4$ (see [16]). 
Remark 2.3. Notice that there exist quantum type representations of $S p(2 g, \mathbb{Z})$, for instance those associated to the monodromy of level $k$ theta functions in the $U(1)$ gauge theory (see e.g. [12, 14]). Again these are only projective unitary representations which lift to unitary representations of some central extension $\rho_{S p, k}$ : $\widetilde{S p(2 g, \mathbb{Z})}(4) \rightarrow U\left(k^{g}\right)$. Here $S \widetilde{S(2 g, \mathbb{Z})}(4)$ is the central extension of $S p(2 g, \mathbb{Z})$ by $\mathbb{Z}$ whose class is 4 times the Maslov class. However, these representations factor through the integer metaplectic group. Further, the generator of the kernel of

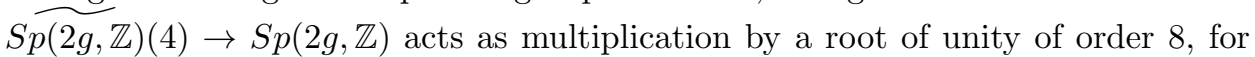
any level $k$. Thus the intersection of $\bigcap_{k \geq 2} \rho_{S p, k}$ is $2 \mathbb{Z}$, and the result of Deligne cited above shows that this is sharp.

\section{Proof of Proposition 1.2}

We consider first the following notion introduced by Farb in $[9$ :

Definition 3.1. Let $n \geq 1$. A group $\Gamma$ has property $F A_{n}$ if any isometric action on any $n$-dimensional $\operatorname{CAT}(0)$ cell complex $X$ has a fixed point.

Observe that property $F A_{1}$ corresponds to Serre's property $F A$, which asks that any action without inversions of $\Gamma$ on a real tree should fix a vertex. Notice that Kazhdan groups have property $F A$. Moreover if a group has property $F A_{n}$, then it has property $F A_{k}$ for all $k<n$. It is known (see 9]) that a group $\Gamma$ with property $F A_{n-1}$ has $n$-integral representation type; namely, the eigenvalues of matrices in $\rho(\Gamma)$, for a homomorphism $\rho: \Gamma \rightarrow G L(n, K)$ with $K$ a field, are algebraic integers if $\operatorname{char}(K)=0$. Moreover, there are only finitely many conjugacy classes of irreducible representations of $\Gamma$ into $G L(n, K)$, for an algebraically closed field $K$.

Culler and Vogtmann proved that $M_{g}$ has property $F A_{1}$ in [5]. In [7] one asks to estimate the maximal $n=n(g)$ for which $M_{g}$ has property $F A_{n}$.

There is a version of $F A_{n}$, namely the strong $F A_{n}$ (which implies $F A_{n}$ ), in which one considers complete $\mathrm{CAT}(0)$ spaces and semi-simple actions. It is proved by Bridson in [3] (see also [2]) that $M_{g}$ has strong $F A_{g}$. Moreover it is known that $M_{g}$ acts (faithfully if $g>2$ ) by semi-simple isometries on the completion of the Teichmüller space with the Weil-Petersson metric, which has dimension $6 g-6$. Thus $g \leq n(g) \leq 6 g-7$.

The key point is to relate the property $F A_{n}$ to the finiteness of unitary representations. Specifically, we have the following:

Proposition 3.1. If $\Gamma$ is a finitely generated group with property $F A_{n^{2}-1}$, then the representations $\Gamma \rightarrow S L(n, \mathbb{C})$ have finite image.

Proof. Let $\bar{\Gamma}$ be the image of $\Gamma$ under some homomorphism into $S L(n, \mathbb{C})$. A finitely generated subgroup $\bar{\Gamma}$ of $S L(n, \mathbb{C})$ lies in some $S L(n, A)$, where $A$ is a finitely generated $\mathbb{Q}$-algebra contained in $\mathbb{C}$. Let $\varphi: A \rightarrow \overline{\mathbb{Q}}$ be a specialization of $A$, which induces a morphism $\varphi: S L(n, A) \rightarrow S L(n, \overline{\mathbb{Q}})$. The image $\varphi(\bar{\Gamma})$ belongs then to some $S L(n, K)$, where $K$ is a finite extension of $\mathbb{Q}$.

Lemma 3.1. If all specializations $\varphi(\bar{\Gamma})$ are finite, then $\bar{\Gamma}$ is finite.

Jordan's theorem says that there is some $f(n)$ such that any finite subgroup of $G L(n, K)$ has a normal abelian subgroup of index at most $f(n)$. The intersection of all subgroups of $\bar{\Gamma}$ of index at most $f(n)$ is then a finite index subgroup $U \subset \bar{\Gamma}$ such that $\varphi([U, U])=1$ for every specialization $\varphi$. Since specializations $\varphi$ separate 
the points of $A$ we have $[U, U]=1$. Therefore there exists a finite index normal abelian subgroup $U$ of $\bar{\Gamma}$. If $U$ is finite, then $\bar{\Gamma}$ will be finite, and we are done.

Let us assume from now on that $U$ is infinite. Since $U$ is finitely generated abelian, there is an infinite order element $Z \in U$. The following lemma will show that there exists a specialization $\varphi$ such that $\varphi(Z)$ is of infinite order, contradicting our assumptions. Thus $U$ should be finite abelian and the result will follow.

Lemma 3.2. Let $Z$ be a matrix with entries in a finitely generated $\mathbb{Q}$-algebra $A$ contained in $\mathbb{C}$. Suppose that for every number field $K$ and any ring homomorphism $\varphi: A \rightarrow K$ the image $\varphi(Z)$ is a matrix of finite order. Then $Z$ has finite order.

Proof. By Noether's normalization lemma (see [19], p. 63) there exist algebraically independent elements $\xi_{1}, \xi_{2}, \ldots, \xi_{p} \in A$ such that $A$ is an integral extension of the purely transcendental extension $B=\mathbb{Q}\left[\xi_{1}, \ldots, \xi_{p}\right]$. Moreover $\xi_{1}, \ldots, \xi_{p}$ form a transcendence basis for the field of fractions of $A$ over $\mathbb{Q}$.

Let $\lambda_{1}, \ldots, \lambda_{n}$ be the eigenvalues of the matrix $Z$. We will prove that $\lambda_{j}$ are roots of unity. First $\lambda_{j}$ are integral over $A$ because they are the roots of the characteristic polynomial of $Z$, which is a monic polynomial with coefficients in $A$. The integrality is transitive and hence $\lambda_{i}$ are integral over $B$. Thus $\lambda_{j}$ satisfies an algebraic equation $P_{j}\left(\lambda_{j}\right)=0$, where $P_{j} \in B[X]$ is the minimal polynomial of $\lambda_{j}$ over $B$. The polynomial $P_{j}$ is monic and irreducible because $B$ is a unique factorization domain. This implies that if we consider $P_{j}$ as a polynomial from $\mathbb{Q}\left[\xi_{1}, \ldots, \xi_{p}, X\right]$, then it is still an irreducible polynomial in the $p+1$ variables $\xi_{1}, \ldots, \xi_{p}, X$.

If $p=0$, then the fractions field of $A$ is a number field and thus the $\lambda_{i}$ should be roots of unity.

Let us assume henceforth that $p \geq 1$. Observe that any specialization $\varphi$ : $B \rightarrow \overline{\mathbb{Q}}$ can be lifted (not uniquely) to a specialization $\varphi: D \rightarrow \overline{\mathbb{Q}}$ of a finite extension $D$ of $B$. First, specializations of $B$ can be extended to possibly infinite specializations (see [20, Thm. 6, p. 31) of any extension $D$ of $B$. Moreover the extended specialization is finite on any element of $D$ which is integral over $B$ (see [20, Prop. 22, p. 41). In particular, any specialization of $B$ extends to $A\left[\lambda_{1}, \ldots, \lambda_{n}\right]$. On the other hand, observe that any specialization $\varphi$ of $B$ corresponds to prescribing the values of $\varphi\left(\xi_{j}\right) \in \overline{\mathbb{Q}}$ arbitrarily.

Hilbert's irreducibility theorem states that there exist infinitely many (actually a Zariski dense set of) specializations $\varphi: B \rightarrow \mathbb{Q}$ such that the polynomials $\varphi\left(P_{j}\right) \in$ $\mathbb{Q}[X]$ are still irreducible. Since $\varphi(Z)$ is of finite order, each $\varphi\left(\lambda_{j}\right)$ is a root of unity so that $\varphi\left(P_{j}\right)$ should be a cyclotomic polynomial. The degree of $\varphi\left(P_{j}\right)$ is the degree $d_{j}$ of $P_{j}$, since these are monic polynomials. But there are only finitely many cyclotomic polynomials of a given degree. Let $S$ be the finite family of coefficients of all cyclotomic polynomials of degree smaller than or equal to $\max \left(d_{1}, \ldots, d_{n}\right)$. It suffices then to choose some specialization $\varphi$ of $B$ for which one coefficient of some $\varphi\left(P_{j}\right)$ does not belong to $S$. For instance it suffices to choose a specialization for which some coefficient of $\varphi\left(P_{j}\right)$ is not in $\mathbb{Z}$, because cyclotomic polynomials have coefficients in $\mathbb{Z}$. This is possible unless all coefficients of the polynomials $P_{j}$ are independent on the $\xi_{1}, \ldots, \xi_{p}$. This might happen only if $P_{j} \in \mathbb{Q}[X]$, namely if all its coefficients, which are elements of $\mathbb{Q}\left[\xi_{1}, \ldots, \xi_{p}\right]$, are actually constant. But in this case all $\lambda_{j}$ are algebraic integers. This contradicts the fact that the transcendence degree of the fractions field of $A$ was supposed to be $p \geq 1$. Therefore all eigenvalues $\lambda_{j}$ of $Z$ are roots of unity. 
An alternative argument is as follows. The set of $\mathbb{C}$-valued specializations $\varphi$ : $A\left[\lambda_{1}, \ldots, \lambda_{n}\right] \rightarrow \mathbb{C}$ is an irreducible affine algebraic variety of dimension $p$ and $\lambda_{j}$ is a rational function on it. If $\lambda_{j}$ is a root of unity for any $\overline{\mathbb{Q}}$-valued specialization, then $\left|\lambda_{j}\right|$ is identically 1 . But a bounded regular function on an irreducible complex algebraic variety should be constant. This implies that all $\lambda_{j}$ are algebraic integers, and we conclude as above.

Eventually, it suffices to show that $Z$ is diagonalizable. Consider the JordanChevalley decomposition $Z=D+N$, where $D$ is semi-simple, $N$ is nilpotent and $D N=N D$. The entries of the matrices $D$ and $N$ belong to the field of fractions of $A$ (see [4, Thm. 7, pp. 71-72). Let $a \in A$ be the least common multiple of denominators arising in the entries of $D$ and $N$. Every specialization $\varphi$ of $A$ with the property that $\varphi(a) \neq 0$ extends uniquely to a specialization, still denoted $\varphi$, of the localization of $A$ at $a$. In particular, it makes sense to consider $\varphi(D)$ and $\varphi(N)$. Therefore $\varphi(Z)=\varphi(D)+\varphi(N)$ is the Jordan-Chevalley decomposition of $\varphi(Z)$. But the minimal polynomial of $\varphi(Z)$ divides $X^{s}-1$, where $s$ is the order of $\varphi(Z)$. This implies that the minimal polynomial has distinct roots and so $\varphi(Z)$ is semi-simple. The uniqueness of the Jordan-Chevalley decomposition yields then $\varphi(N)=0$. Since this holds for any specialization $\varphi$ such that $\varphi(a) \neq 0$ and such specializations separate the points of $A$, we derive that $N=0$. Thus $Z$ is diagonalizable and hence of finite order, as claimed.

Remark 3.1. We could also use ([3], Prop. 6.1), which says that the image in $G L(g, \mathbb{C})$ of an element of a finitely generated group with strong property $F A_{g}$ has algebraic eigenvalues. However, Lemma 3.2 can be applied to more general situations, since there is no assumption on $Z$.

It suffices now to show that for any specialization $\varphi$, the image $G=\varphi(\bar{\Gamma})$ is finite. Observe that if $\Gamma$ has property $F A_{n-1}$, then $G=\varphi(\bar{\Gamma})$ has also property $F A_{n-1}$. We will show that:

Lemma 3.3. Let $K$ be a number field. Then a finitely generated subgroup $G \subset$ $S L(n, K)$ with property $F A_{n^{2}-1}$ should be finite.

Proof. We prove that for any embedding of $K$ into a local field $K_{v}$ the image of $G$ in $S L\left(n, K_{v}\right)$ is precompact.

If $G \subset S L(n, K)$ is a finitely generated subgroup with property $F A_{n-1}$, then its image in $S L\left(n, K_{v}\right)$ is precompact for each non-Archimedean valuation $v$ of $K$. In fact, $G$ acts on the Bruhat-Tits building associated to $S L\left(n, K_{v}\right)$, which is an $(n-1)$-dimensional CAT $(0)$ cell complex. The $G$-action has a fixed point because $G$ has property $F A_{n-1}$, and hence $G$ is contained in the stabilizer of a vertex, which is a compact subgroup.

In what concerns the Archimedean valuations it suffices to consider the complex ones. But $S L(n, \mathbb{C})$ acts on the symmetric space $S L(n, \mathbb{C}) / S U(n)$ of non-compact type and real dimension $n^{2}-1$. Since this space is $\operatorname{CAT}(0)$ and $G$ has property $F A_{n^{2}-1}$, it follows that the image of $G$ into $S L(n, \mathbb{C})$ is contained in the stabilizer $U(n)$ for any complex valuation inducing an embedding $K \rightarrow \mathbb{C}$.

Eventually recall that $S L(n, K)$ embeds as a discrete subgroup of the special linear group $S L\left(n, A_{K}\right)$ over the adèle ring $A_{K}$ of $K$. By the above $G$ is discrete and precompact into $S L\left(n, A_{K}\right)$ and hence finite.

This proves Proposition 3.1 . 
Remark 3.2. If $G$ is a subgroup of $U(n) \cap S L(n, \mathbb{Q})$ with property $F A_{n-1}$, then $G$ is finite. This follows from the above by using the fact that there is a unique complex Archimedean valuation on $\mathbb{Q}$, and one knows already that $G$ is contained in the compact group $U(n)$. In particular, if $\Gamma$ has property $F A_{n-1}$, then the image of any homomorphism $\Gamma \rightarrow U(n) \cap S L(n, \mathbb{Q})$ is finite.

End of the proof of Proposition 1.2. The result of Proposition 3.1 holds also for representations into $P S L(n, \mathbb{C})$ and a fortiori for representations into $P U(n)$. Since $M_{g}$ has property $F A_{g}$ we derive the lower bound inequality.

Consider now the smallest (projective) quantum representation $M_{g} \rightarrow P U\left(d_{g}\right)$ with infinite image, for $g \geq 2$. This is the $S O(3)$ quantum representation in level 5 (see e.g. [13]), whose dimension $d_{g}$ is given by the Verlinde formula:

$$
d_{g}=\left(\frac{5}{4}\right)^{g-1} \sum_{j=1}^{4}\left(\sin \frac{2 \pi j}{5}\right)^{2-2 g}=\left\{\begin{array}{l}
5^{g / 2} F_{g-1}, \text { if } g \text { is even, } \\
5^{(g-1) / 2}\left(F_{g}+F_{g-2}\right),
\end{array}\right.
$$

where $F_{j}$ is the Fibonacci sequence $F_{0}=0, F_{1}=1, F_{n+1}=F_{n}+F_{n-1}$, for instance $d_{2}=5$. These mapping class group representations come from the so-called Fibonacci TQFT.

Moreover, it is clear that the upper bound holds for any finite index subgroup of $M_{g}$. In fact the image of a finite index subgroup of the mapping class group by the quantum representation is still infinite. This proves the claim.

Remark 3.3. The property $F A_{n-1}$ is not inherited by the finite index subgroups. Actually $M_{2}$ has a finite index subgroup which surjects onto a free non-abelian group and hence it does not have property $F A_{1}$. The situation is subtler for $g \geq 3$, and it seems unknown whether finite index subgroups of $M_{g}$ have property $F A_{1}$. Bridson proved in [2] that for any normal subgroup $H$ of index $n$ in $M_{g}$, for $g \geq 3$, and any homomorphism $\phi: H \rightarrow G$ to a group $G$ acting by hyperbolic isometries on some complete $\operatorname{CAT}(0)$ space (in particular, to $G=\mathbb{Z}$ ) the $n$-th powers of Dehn twists (which belong to $H$ ) lie in the kernel of $\phi$. Such homomorpisms $\phi$ have therefore striking similarities with the quantum representations.

Corollary 1.1 follows from Proposition 3.1 above and Bridson's result from [3] saying that $M_{g}$ has strong $F A_{g}$.

\section{ACKNOWLEDGEMENTS}

We are indebted to Jean-Benoît Bost, Benson Farb, Eric Gaudron and Bertrand Remy for helpful discussions and to the referees for pointing out an incomplete argument in the first version. The author was partially supported by the ANR Repsurf: ANR-06-BLAN-0311.

\section{REFERENCES}

[1] J. E. Andersen, Asymptotic faithfulness of the quantum $\mathrm{SU}(n)$ representations of the mapping class groups, Ann. of Math. (2) 163(2006), 347-368. MR2195137(2007c:53131)

[2] M. R. Bridson, Semisimple actions of mapping class groups on CAT(0) spaces, arXiv:0908.0685, "The Geometry of Riemann Surfaces", London Mathematical Society Lecture Notes, 368, dedicated to Bill Harvey on his 65th birthday, to appear.

[3] M. R. Bridson, On the dimension of CAT(0) spaces where mapping class groups act, arXiv:0908.0690.

[4] C. Chevalley, Théorie des groupes de Lie II. Groupes algébriques, Actualités Sci. Ind. 1152, Hermann \& Cie., Paris, 1951. MR0051242 (14:448d) 
[5] M. Culler and K. Vogtmann, A group-theoretic criterion for property FA, Proc. Amer. Math. Soc. 124(1996), 677-683. MR.1307506 (96f:20040)

[6] P. Deligne, Extensions centrales non résiduellement finies de groupes arithmétiques, C. R. Acad. Sci. Paris Sér. A-B 287(1978), no. 4, A203-A208. MR507760 (80g:20056)

[7] B. Farb, Some problems on mapping class groups and moduli space, in Problems on mapping class groups and related topics, 11-55, Proc. Sympos. Pure Math., 74, Amer. Math. Soc., Providence, RI, 2006. MR2264130 (2007h:57018)

[8] B. Farb and D. Margalit, A primer of mapping class groups, preprint.

[9] B. Farb, Group actions and Helly's theorem. Advances Math. 222(2009), 1574-1588. MR2555905

[10] B. Farb, A. Lubotzky and Y. Minsky, Rank one phenomena in mapping class groups, Duke Math. J. 106(2001), 581-597. MR1813237 (2001k:20076)

[11] M. H. Freedman, K. Walker and Zhenghan Wang, Quantum SU(2) faithfully detects mapping class groups modulo center, Geom. Topol. 6(2002), 523-539. MR.1943758 (2004a:57037)

[12] L. Funar, Représentations du groupe symplectique et variétés de dimension 3, C. R. Acad. Sci. Paris Sér. I Math. 316(1993), no. 10, 1067-1072. MR1222974 (95g:57030)

[13] L. Funar, On the TQFT representations of the mapping class groups, Pacific J. Math. 188(1999), 251-274. MR.1684208 (2000d:57026)

[14] L. Funar, Some abelian invariants of 3-manifolds, Rev. Roumaine Math. Pures Appl. 45(2000), 825-861. MR 1865997 (2003f:57023)

[15] S. Gervais, Presentation and central extensions of mapping class groups, Trans. Amer. Math. Soc. 348(1996), 3097-3132. MR1327256 (96j:57016)

[16] M. Korkmaz and A. I. Stipsicz, The second homology groups of mapping class groups of oriented surfaces, Math. Proc. Cambridge Philos. Soc. 134(2003), 479-489. MR.1981213 (2004c:57033)

[17] G. Margulis, Discrete subgroups of semisimple Lie groups, Ergebnisse Math. Grenz. 17, Springer-Verlag, Berlin, 1991. MR,1090825 (92h:22021)

[18] G. Masbaum and J. D. Roberts, On central extensions of mapping class groups, Math. Ann. 302(1995), 131-150. MR 1329450 (96i:57013)

[19] M. Reid, Undergraduate commutative algebra, London Mathematical Society Student Texts 29, Cambridge University Press, Cambridge, 1995. MR.1458066 (98c:13001)

[20] A. Weil, Foundations of Algebraic Geometry, American Mathematical Society Colloquium Publications 29, American Mathematical Society, New York, 1946. MR0023093 (9:303c)

Institut Fourier, BP 74, UMR 5582, University of Grenoble I, 38402 Saint-MartinD'HÈres CEDEX, France

E-mail address: funar@fourier.ujf-grenoble.fr 